\title{
SPRINTING OUT OF STUCKNESS: OVERCOMING MOMENTS OF STUCKNESS TO SUPPORT THE CREATIVITY FLOW IN AGILE TEAM SETTINGS
}

\author{
Shah, Ashni; Huidobro Pereda, Alfonso; Gonçalves, Milene \\ Delft University of Technology
}

\begin{abstract}
Multidisciplinary agile teams working in fast paced, delivery-oriented sprint cycles of two weeks can experience moments of stuckness. Typically, these moments can be characterised by the inability to continue, which can be quite detrimental in agile settings, where time is pressured. This paper aims to explore these moments of stuckness, to understand when and why they occur and to understand the different strategies Scrum teams use to overcome them, both on a personal as well as team level. A combination of interviews and observations were conducted with six Scrum team members and two experts to understand their process and experiences while working in an agile set up. We have identified five strategies, which strongly rely on agile values of collaboration, communication, and creativity. These are: looking for expert guidance, open communication, creating spike stories, visual communication and incubation. The findings from this study provide both practice and academia with a deeper understanding into how can creativity be supported in agile settings.
\end{abstract}

Keywords: Creativity, Design process, Teamwork, Agile, Scrum

Contact:

Shah, Ashni Devan

Delft University of Technology

Industrial Design Engineering

The Netherlands

ashni13@gmail.com

Cite this article: Shah, A., Huidobro Pereda, A., Gonçalves, M. (2019) 'Sprinting Out of Stuckness: Overcoming Moments of Stuckness to Support the Creativity Flow in Agile Team Settings', in Proceedings of the 22nd International Conference on Engineering Design (ICED19), Delft, The Netherlands, 5-8 August 2019. DOI:10.1017/dsi.2019.241 


\section{INTRODUCTION}

Agile is an approach that aims to deliver early and continuous value throughout the duration of a project by means of iterative and incremental developments (Fowler and Highsmith, 2001). It was initially devoted to software development and was born with the aim of promoting collaboration, minimising unnecessary work, engaging customers and stakeholders and accepting uncertainty (Dingsøyr et al., 2012). Since the Agile manifesto was developed in 2001 (Fowler and Highsmith, 2001), the approach has been adopted by many other disciplines and multidisciplinary teams. Design is one of the fields where Agile has been heavily embraced.

There are several Agile process frameworks such as XP, Kanban and Scrum (Jongerius, 2012). In this paper we will focus on Scrum, as it is the most commonly used framework. The official Scrum guide defines it as a framework, based on sprints (short periods of work, usually two weeks), wherein quick design cycles occur (Sutherland and Schwaber, 2013). Each sprint cycle is divided into small tasks, called user stories, which are ranked in order of priority. The goal of each sprint is to complete a set of user stories to deliver a shippable part of the final product at the end of each cycle, culminating in a reflection on the overall process and results (Figure 1).

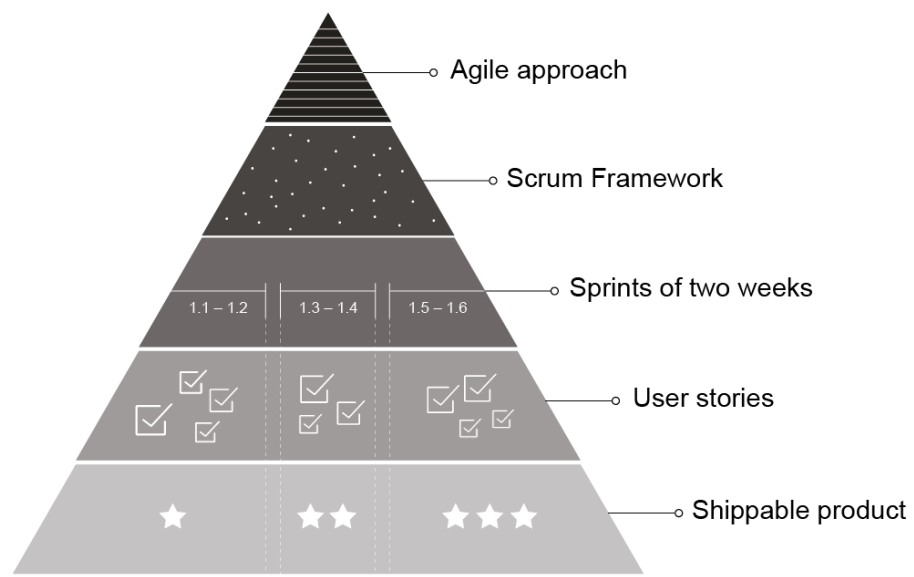

Figure 1. Overview of the scrum process framework

Although Agile and Scrum aim for highly effective and productive processes, with continuous cycles of ideation, validation and iteration, standstills can occur. More specifically, during sprint cycles, team members sometimes experience they feel stuck or fixated, as they are required to iterate and reframe creatively in every sprint (Conboy, Wang and Fitzgerald, 2009). Such cognitive hindrances have often been clustered into the group of phenomena related to design fixation (e.g., Crilly and Cardoso, 2017). In the most general terms, design fixation has been described as "a state in which someone engaged in a design task undertakes a restricted exploration of the design space due to an unconscious bias resulting from prior experiences, knowledge or assumptions" (Crilly and Cardoso, 2017, p.6). A similar concept to fixation is stuckness (Sachs, 1999; Gonçalves, 2017), which encompasses many types of creative hindrances that ultimately can be categorized as "not knowing how to continue". In a fast-paced process such as Scrum, "not knowing how to continue" can be particularly detrimental. However, far too little attention has been paid to this issue.

This paper aims to understand the flow of creativity in multidisciplinary Agile teams and to learn more about how these teams cope with moments during which they are unable to come up with new ideas or directions, making them feel stuck. The research is conducted by observing and interviewing practitioners and experts that work in Agile settings. In the following sections we discuss the existing literature around Agile, Scrum and obstacles to creativity, and propose our research question.

\section{LITERATURE REVIEW}

The Agile approach thrives on the ability of multidisciplinary teams to work cohesively. At its core, Agile approaches are people-centric and they are based on the social paradigm of collaboration (Whitworth and Biddle, 2007). Another pillar for the Agile approach is to work in sprints and deliver 
potentially shippable products at the end of each cycle (e.g., Dick et al., 2013). A potentially shippable product is composed by the sum of the product backlog items delivered in each sprint and can take many shapes, such as a new feature in a mobile application. The fast pace of deliverables required in sprints carries the underlying assumption that creativity is highly expected in order to produce value. Hence, it is important to investigate creativity in the Agile and Scrum contexts, to support the flow of work and ability of a team to deliver during each of these stages (Conboy, Wang and Fitzgerald, 2009). The literature review is clustered into three categories, which are deemed essential for the context of Agile and Scrum - collaboration, communication and creativity.

\subsection{Collaboration}

Even though Agile greatly relies on collaboration (Whitworth and Biddle, 2007), multidisciplinary collaboration is often challenging, since all team members come with their own set of preconceived notions and frames. These frames are the mental models individuals have, which stem from their belief systems, values, and goals; in sum, an individual's worldview (Nonaka, 1991).

Lindsjørn et al. (2016) stated that team members tend to rate the team's performance highly if the team has been engaging in healthy internal processes such as effective collaboration and conflict resolution. Therefore, when team members put in effort to understand the perspective of others, it becomes easier for the team to align their frames and be on the same page, to help improve the quality of teamwork in people-centric Agile settings (Lindsjørn et al., 2016).

\subsection{Communication}

Besides collaboration, communication is key in Agile teams (Whitworth and Biddle, 2007). In order to deliver early and continuous value (Fowler and Highsmith, 2001), Agile teams are set up in a manner that allows all individual team members to feel empowered and have a great sense of ownership and responsibility for their work (Whitworth and Biddle, 2007). Throughout a sprint, team members have four types of scheduled meetings: planning, backlog refinement, review and retrospective (Figure 2). During these sessions the team members communicate the status of their work and share updates on the different tasks and user stories that they have been working on. These meetings are also used as moments to reflect on individual and team performances and plan future sprints.

Hence, we can see that in this set up, communication is facilitated not only verbally, but also by physical and geographical proximity. This is why teams meet daily for stand-ups and also work together from the same floor or space in an office (James and Walter, 2016). Because Agile teams never work in silos, they are able to communicate frequently and collaborate efficiently, eventually resulting in a better quality output (Whitworth and Biddle, 2007).

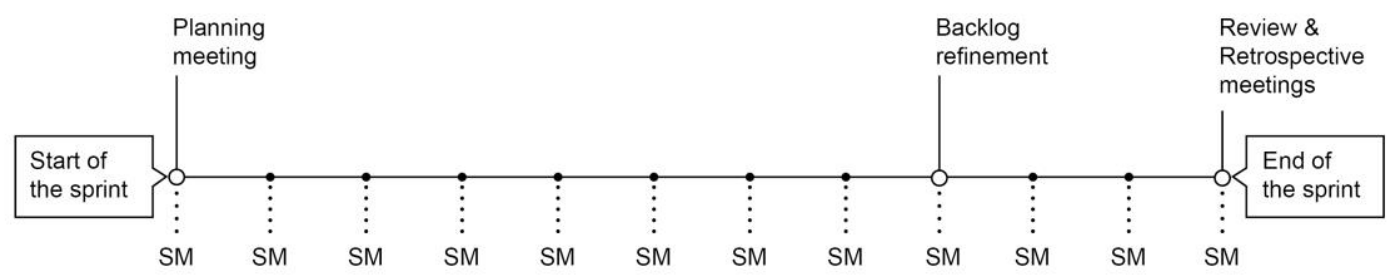

SM: Standup meeting or Daily Scrum

Figure 2. Overview of the meetings within a sprint

\subsection{Creativity}

A review on creativity conducted by Hennessey and Amabile (2010) concluded that according to most researchers creativity entails the creation of solutions that convey value to one or to many. One can then create a parallel with Agile, as the delivery of early and continuous value is a strong driver for the Agile approach (Fowler and Highsmith, 2001).

In Scrum, the development of user stories during a sprint follow a cyclic process that we describe as ideate-validate-iterate. A user story is a set of requirements which is often divided into smaller tasks (Lindsjørn et al., 2016). A sprint typically begins with an ideation phase, followed by validation, in which the developed concept is tested. It is then followed by an iteration phase during which the concept is often refined based on testing or even ideated from scratch. This process provokes a feeling 
of uncertainty in the team, which is coped with by relying on the team's creativity and interactions rather than on processes and tools (Cockburn, 2001). This cycle can often be exhausting for the team, due to time constraints and the need to be continuously creative (Conboy, Wang and Fitzgerald, 2009). Dorst and Cross (2001) state that irrespective of whether it can be classified as a "big" or "small" one, every design process has creative moments. However, to the extent of our knowledge little attention has been paid to how much creativity is involved specifically in Agile processes and how can it be supported to avoid moments of stuckness.

To address this gap, we believe that Amabile's three componential model of creativity (1983) can be helpful. This model indicates that creativity is influenced by: domain relevant skills (the expertise in a particular domain where the problem-solver is working); creativity relevant skills (the cognitive and personality processes conducive to novel thinking); and task motivation (the intrinsic motivation to engage in the activity out of interest, enjoyment, or a personal sense of challenge). As Scrum is a particularly collaborative approach, with a multitude of disciplines involved, this particular model (Amabile, 1983) seems to be an appropriate framework to explore creativity (or the lack of it) in the Agile context.

\section{RESEARCH QUESTION}

From the literature review it is evident that Agile approaches - such as Scrum - focus and rely on a team's ability to be creative on a recurrent basis through the duration of the sprint. And it is this link between creativity and Agile processes that we would like to elaborate on in our paper, since so little research has been found on the connection between these two topics. Considering that there is a need to be continuously creative (Conboy, Wang and Fitzgerald, 2009), the research question we propose is: "How do Scrum teams cope with potential moments of stuckness to support the flow of creativity?"

\subsection{Academic and practical relevance}

A surprising limited number of studies have explored the relationship between creativity and Agile processes. Conboy et al. (2009) studied the role of creativity within the Agile systems development, but most other existing research addresses both topics in silos. This study will contribute to the understanding of the dependencies and influences of the two aspects on each other, more specifically about what obstacles to creativity team members encounter and how they overcome those.

The Agile paradigm has been welcomed by companies around the world, as it provides a way to survive in an unpredictable and rapidly changing market (Denning, 2016; Highsmith and Cockburn, 2001). In this way, big companies such as Ericsson, Microsoft, Barclays and Spotify have embraced the Agile approach and become pioneers of innovation in their respective domains. The term creative economy, closely grounded in the Agile approach, reinforces then its fundamental principle (Denning, 2016). A creative economy is focussed on the sustained generation of new products and services, the success of which depends on continuous innovation and the delivery of additional value to customers for which they are willing to pay. In this context, it is paramount to research how can we support creativity in Agile settings.

To conclude, this paper aims to explore the episodes when Agile, more specifically, Scrum teams experience stuckness and lack of creativity during sprints, in order to understand why these moments occur, how teams cope and provide insights to support the creative process in Agile settings.

\section{METHOD}

In order to answer our research question, we chose a qualitative approach. Particularly, we have gathered insights from professional designers and experts on their experiences and practices through interviews and observations. These are divided in two parts, which are explained below: practice focused and expert focused. A qualitative approach was considered appropriate, as it enables to study the context of events, its different conditions and the relationship between them (Dooley, 2002).

\subsection{Interviews and observation}

With the combination of interviews and observations we aim to understand real life situations while retaining the richness of the context and meaning of the people involved (Yin, 2009). On one hand, interviews are useful because they help uncovering information that cannot be directly observed, such as experiences, motivations and value systems of individual participants. On the other hand, 
observations help to identify contradictions and misalignment between verbal and nonverbal behaviour of participants (Patton, 2002), in their natural settings. For the purpose of this study, we used observations as a secondary method to enhance our primary research method of interviews.

\subsection{Practice focus}

In the first part of our research, we interviewed and observed two multidisciplinary teams in two different organizations, with the aim of capturing the real experiences of working in Agile settings. We also observed one team during the planning meeting for 1.5 hours. Data obtained from these observations was not directly useful for our research since the planning meeting is not a "creative" moment in the Scrum process. However, since this is a meeting which involves discussions among the entire team, it was useful for us to understand the team dynamics and overall process and to support the main findings from the interviews.

For the interviews, we pre-selected three to four team members from each organization, based on their role in the team. We preferred to interview those who are more involved in the creative parts of the process, such as the product owners, Scrum masters, interaction or user experience designers and managers.

\subsection{Expert focus}

The interviews with the experts were meant to validate the findings from the practice focused data collection part. Interviews were conducted with two experts in the field of Agile and Scrum practices.

\subsection{Sample selection}

The practice-focused part consisted of a set of 8 interviews and one observation session conducted at Werkspot (a Dutch marketplace for hiring services from service professionals such as plumbers or painters) and at ING Bank. We conducted interviews with four team members (at Werkspot) and two (at ING Bank). In both cases, the participants were working in multidisciplinary teams using the Scrum process framework. The following profiles were selected: UX Designer, Scrum Master, Product Owner, Project Manager and Front-End Developer. Due to confidentiality issues, Werkspot was the only company where we conducted an observation session. We did not control for gender balance. The available and most suitable participants were male (5 out of 6 ).

The expert-focused part consisted of interviews with two experts in the field of Scrum practices Pieter Jongerius, who is Director at Fabrique and author of the book "Get Agile!"; and Guilherme Uhelski who is a Scrum Master at Werkspot with 10 years of experience in the role. Interviews with the experts were conducted as a way to verify our findings and bridge the gap between them and research question. Figure 3 presents an overview of the participant profiles.
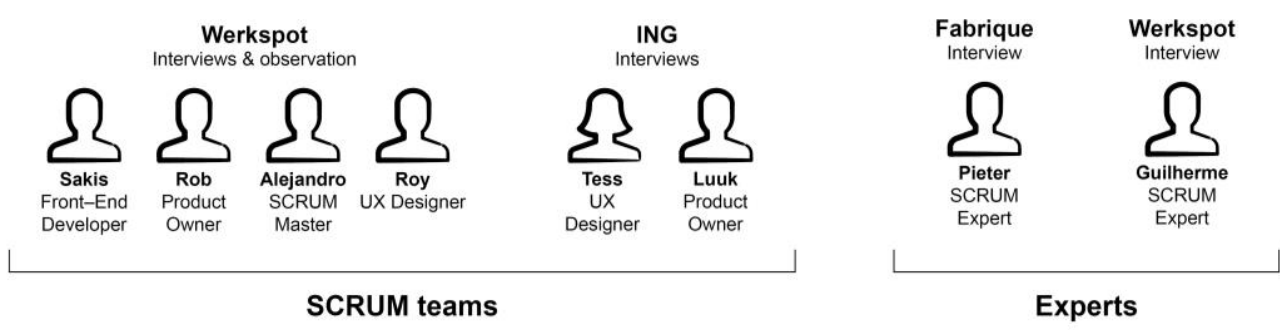

Figure 3. Overview of participants

\subsection{Interview guide}

We used observation and interview guides to conduct the sessions. This helped to increase the comprehensiveness of the data and made the process of data collection systematic across participants (Patton, 2002). The sub topics covered during the interview were:

- Understanding the design process

- Understanding the moments of stuckness

- Understanding personal strategies for overcoming moment(s) of stuckness

- Understanding team-level strategies for overcoming moments of stuckness 


\subsection{Data analysis}

All the interviews were audio taped and transcribed. The observation material was used to corroborate whether the behaviour of the interviewees during team work was in line with what they express during the interviews. From the transcripts we extracted in-vivo codes, which helped us retain the participant's context. From these initial codes, it was possible to cluster them into further categories and themes, according to our research question.

We performed the categorization on three transcripts together, and after agreeing on the definitions of the different codes we performed categorization on three transcripts individually. By means of this we tried to establish partial inter-rater reliability by manually comparing the different categories and arrived at an agreement of $70 \%$.

Figure 4 shows the resulting categories in relation to the themes, which are used to guide the discussion.

\begin{tabular}{|c|l|}
\multicolumn{2}{|c|}{ Themes } \\
\hline \multirow{3}{*}{ Moments of creativity } & Process characteristics \\
& Process phases \\
& Bridging problem space \\
\hline \multirow{3}{*}{ Moments of stuckness } & Communication \\
& Team activities \\
& Team qualities \\
& Individual qualities \\
\hline Unblocking stuckness \& & Personal strategies \\
Future advice & Team strategies \\
\hline
\end{tabular}

Figure 4. Overview of categories

\section{RESULTS}

In this section we explain our findings in relation to the research question. We mention the different themes according to our insights, supported by quotes from participants and experts.

\subsection{Understanding the design process}

According to literature, the Scrum methodology employs an iterative and incremental approach in order to optimize and control risk during the sprint cycle (Sutherland and Schwaber, 2013). In our interviews analysis, we have found that, in Scrum teams, uncertainty is dealt by relying on people and their creativity for problem-solving, rather than on a sacrosanct process. This is done by use of tools/techniques that help to generate appropriate practices for different situations (Highsmith and Cockburn, 2001). Characteristics of the Scrum process, such as daily stand-ups, retrospective sessions and use of open workspaces, facilitate the team to work cohesively towards a larger common goal. In our interviews, the Scrum expert Pieter Jongerius stated that "Scrum is a process aimed at progress". Hence, building consistency in the process and integrating these characteristics into the routine of all team members helps them to focus on creating the solution rather than getting distracted by the process, as supported by the following quote:

"The more similarities (in the process) the better it is. The less time we invest in the process the more time we have to work. I try to have the meetings in the same day of the week, at the same time and in the same room." - Alejandro, Scrum Master, Werkspot

Guilherme Uhelski, the Scrum Master expert, mentioned that in order to prevent team members from struggling with tasks during the sprint, it is important to get everyone to understand how Agile works, "not just steps of the process but the deeper level Agile values". The values mentioned here are those included in the Agile Manifesto, such as "individuals and interactions over processes and tools"; "customer collaboration over contract negotiation"; and "responding to change over following a plan" (Fowler and Highsmith, 2001). The expert Pieter Jongerius also agrees: "If you see a Scrum team struggling it helps to look back at the Agile principles such as valuing people over processes, working towards the end goal etc". In practice, these principles seem to be the first support system, to help team 
members to work more efficiently and collaborate more effectively. It ensures that the team is aligned by means of openly discussing and communicating ideas as well as problems and difficulties. In turn, this keeps the team focused on the goal at hand and ensures that everyone on the team is working towards it. Most participants stated iteration occurs in Scrum: ideas do evolve during the sprint as they go through the different phases of ideate-validate-iterate. However, while the different phases of the process play a role and influence the behaviour of an individual and the overall team, the larger influence comes from the ability of each team member to cope with the challenges of iterating during a sprint.

"Scrum is to get products out that work. Pivoting hurts the IT (guys). However designers are more used to pivoting. Rethinking is easier than rebuilding it." - Luuk, Product Owner, ING

While some disciplines within the team are no stranger to accepting the need to change direction (or pivoting, the term preferred in Scrum), our participants indicated that there are struggles in doing so. When their work practices are so ingrained in a routine of sprint cycles, pivoting is an essential but demanding moment. Pivoting seems to be even more important in moments of stuckness.

\subsection{Understanding the moments of stuckness}

According to Lindsjørn et al. (2016), in Agile teams the frequency and formalization of communication matters the most. Frequency is how often team members communicate, while formalization is the degree of spontaneity in their communication (Lindsjørn et al., 2016). In agreement with literature, all participants agreed with the notion that open communication accelerates knowledge sharing, which can be relevant to all team members for tasks during the sprints. More importantly, some interviewees (Scrum Masters and Product Owners) mentioned that one of their main roles in the team was to synchronize team activities and dependencies in order to keep the team on the same page, as supported by Lindsjørn et al. (2016). This helps the team to be aligned and contributes to the quality of teamwork as well as to the success of a project (Whitworth and Biddle, 2007).

"And once per day in the morning we do the stand-up meeting where we synchronize. Something that we do, is that we set daily goals for each one of us, which means that today I will try to achieve this." - Alejandro, Scrum Master, Werkspot

Alejandro, Scrum Master at Werkspot, stated that his main role was to resolve conflicts in the team, identified by him as one of the key elements to achieve high quality teamwork. Ensuring that all team members are aligned helps with the task at hand. However, being aligned is not always a synonym of success. Even aligned, the team can feel stuck when the initial idea or approach does not work and they do not have a back-up plan. Rob, Product owner at Werkspot mentioned that having a plan B is needed in order to help the team overcome moments of stuckness.

"Because on the daily meetings you guys were probably telling me 'okay we try this and it didn't work' then I think we will already have a plan B on our sleeves or something like that" -

Guilherme, Scrum expert, Werkspot.

All participants were of the opinion that Agile processes are people-centric (Whitworth and Biddle, 2007) and by collaborating and working together they can achieve the tasks set for every sprint and make the project a success. Tess, UX Designer at ING, stated that for her it was of paramount importance to be aware of the plan of her team members, due to team dependencies.

"Not knowing this makes the process unstructured, resulting in a feeling of stuckness and makes me kind of frustrated." - Tess, UX Designer, ING

Therefore, it seems that moments of stuckness in Scrum teams are strongly associated with (lack of) communication and alignment between team members.

\subsection{Understanding strategies for overcoming moment(s) of stuckness}

Conboy, Wang and Fitzgerald (2009) stated that, in order for ownership of work to actually enhance creativity, team members must feel like they are working in a non threatening environment, one that is built on trust and openness, where they know it is safe for them to share their ideas and way of doing things. This was a recurrent theme that transpired from the interviews, as seen in the following quote: "People should be aware of how important it is to be transparent in an Agile team." (Guilherme, Scrum expert, Werkspot). 
Most team members spoke about asking for help in situations where they felt stuck. Five strategies emerged from the insights collected, which were indicated by the participants and experts as ways to overcome moments of stuckness:

1. Seeking support from the Scrum master and product owner. Their expertise and their focus on the larger picture are considered to be valuable when encountering standstill moments.

"Sometimes you need escalate the problem. Go to the manager and tell him you are having this problem." - Alejandro, Scrum Master, Werkspot.

2. Reaching out to other colleagues within the company or industry experts that have experience with the matter at hand, and to obtain a fresh perspective.

"So we approach the other teams that are focusing on this to help us. We ask about the difficulty we have and they suggest us to research on other technologies that can do the job and solve our issues." - Alejandro, Scrum Master, Werkspot

3. Planning spike stories, which as one of the Scrum experts defines them - are stories to quickly experiment for the feasibility of a concept or technology. They provide a time-box where experimentation is allowed, to search for alternatives or to overcome perceived stuckness. To some extent, this can be connected to the concept of experimental spaces (Buchner and Langley, 2016), as a way to break away from pre-existing ideas in organisations.

"In those cases is best to do a spike story: an experimental block of time. But the team should get into this knowing fully that if the experiment doesn't work then they have to throw it away"

- Pieter, Scrum expert, Fabrique

4. Using visual communication techniques, since many team members reported they represent their own thoughts through quick sketches or diagrams during meetings to explain their idea more clearly.

"People don't need...so you visualize. You show the first, second and third step and then you actually passed across the message that you want." - Sakis, Front end developer, Werkspot

5. Adopting a detachment attitude, in order to let the idea/problem sink in and then look at it after some time with a refreshed perspective. This is related to incubation, which Wallas (1926, p.86) described as moments "where we do not voluntarily or consciously think on a particular problem". One of the Scrum experts stated that "sometimes all it takes is to get rid of some of the constraints and you will be back in the game."

"To overcome the moments of stuckness, first I do an analytical review of why it didn't work. Then I go home and next day I can start again. Analysing your failure and learning from it, doesn't happen in the same day. Sleeping helps." - Luuk, Product Owner, ING.

\section{DISCUSSION}

In general, all participants reported different instances of experiencing moments of stuckness during the two-week sprints they follow while working with the Scrum framework. From the interviews, it is clear that though participants experienced these moments of stuckness, they have also inherently developed strategies or coping mechanisms to get over them and move forward with the task at hand. In the previous section we reported our findings about five strategies used by participants. Besides these strategies, we found that Scrum is designed in a way that helps teams mitigate moments of stuckness by relying on the team, communicating more frequently and always ensuring that the team is aligned by means of daily stand-up meetings (Whitworth and Biddle, 2007). This is the reason why, when a team is new, it takes some time before they can start delivering successful sprints.

"When you create a new team, it takes some time. If you take a good designer and a good developer and make a team, it will still take some months before they start to work well together." - Alejandro, Scrum Master, Werkspot. 
During the planning meeting, each team member has the liberty to estimate how much time and effort a particular task will take them (Highsmith and Cockburn, 2001), which is followed by a negotiation and agreement among everyone within the team. This seems to lead to a sense of commitment and ownership for each individual team member, which acts as a driving force for them through the duration of a sprint - pushing them to live up to what they committed to. This individual and collective sense of responsibility can make them more proactive in seeking out solutions to problems or resolving moments of stuckness that they might experience during the sprint. Figure 5 illustrates the major insights we have gathered with our study, in relation to the research question: How do Scrum teams cope with potential moments of stuckness to support the flow of creativity? While the top part of the mindmap refers to the characterisation of these moments and why they might occur, the bottom part addresses the main coping strategies on a personal and team level. More importantly, the mindmap establishes possible connections between these coping strategies, strengthening the importance of four factors: relying on Agile values; on collaboration; on communication; and allowing for incubation. Overall, the participants did not seem to have structured approaches or concrete guidelines to deal with stuckness, but were nevertheless able to overcome it (Gonçalves, 2017), by relying on the experience of working as Agile teams.

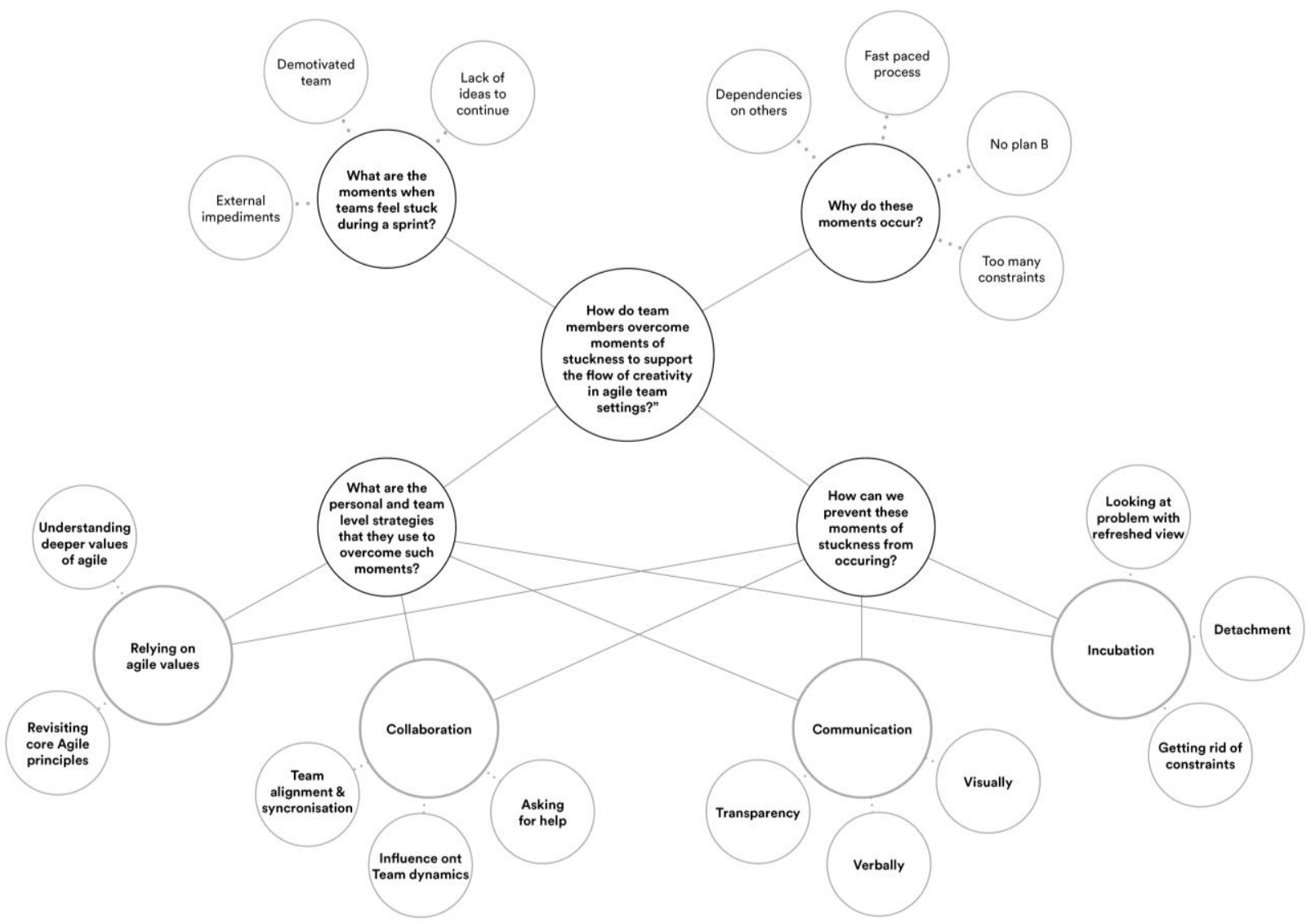

Figure 5. Mindmap of insights regarding the research question

\section{CONCLUSION}

This paper explored, in a qualitative approach, how do Scrum teams cope with potential standstills in their creative process, which we refer to as moments of stuckness. Through the analysis of observations and interviews, we have identified the most important factors that may lead to stuckness in Scrum teams, but also five main coping strategies, applied at the personal and team level, by our participants.

As it is usual, there are a number of potential limitations to be considered, but also opportunities for future research. We acknowledge that our results are based on interviews with two experts and two Scrum teams only. This implies that further research needs to be conducted in order to verify these results and arrive at a theoretical saturation in accordance to the research question. A limitation of our method approach is that it was not possible to observe a team at ING due to strict confidentiality 
clauses at the bank. Hence observing and interviewing more teams in the future would add great value to understanding how can we support Agile teams in their creative process.

In general, qualitative research generates a large amount of data and can be very time consuming to analyse (Gray, 2014). A limitation of conducting interviews is that interviewees tend to give answers which they think the researcher is looking for. As such it could result in biased or incomplete data (Alshenqeeti, 2014). However, we expect that observations helped us to probe deeper into areas which seemed contradictory.

Finally, conducting further research on this topic would also make it possible to propose guidelines to help multidisciplinary Agile teams overcome these moments of stuckness and support the flow of creativity to speed up the process and improve the quality of work.

The insights gathered in this study have a number of implications for practice and research. For practice, this research can impact the development of the Agile approach itself and, in particular, Scrum. Considering the extreme adoption of this approach in the industry, it is critical to reflect and iterate on what the Agile principles are, in order to maintain its perceived values. Regarding research, as no previous literature has been found on how to deal with moments of stuckness, this study can open a new path for academics to investigate which creative challenges teams may experience when adopting the Agile approach and how to cope with them efficiently.

\section{REFERENCES}

Alshenqeeti, H. (2014), "Interviewing as a Data Collection Method: A Critical Review”, English Linguistics Research, Vol. 3 No. 1.

Amabile, T. (1983), “The social psychology of creativity: A componential conceptualization”, Journal of Personality and Social Psychology, Vol. 45 No. 2, pp. 357-376.

Bucher, S. and Langley, A. (2016), "The Interplay of Reflective and Experimental Spaces in Interrupting and Reorienting Routine Dynamics”, Organization Science, Vol 27 No. 3, pp. 1-20.

Conboy, K., Wang, X. and Fitzgerald, B. (2009), "Creativity in Agile systems development: a literature review", Information Systems-Creativity and Innovation in Small and Medium-Sized Enterprises, pp.122-134.

Crilly, N. and Cardoso, C. (2017), "Where next for research on fixation, inspiration and creativity in design?", Design Studies, No. 50, pp.1-38.

Dick, M., Drangmeister, J., Kern, E. and Naumann, S. (2013), “Green software engineering with Agile methods”, In: Green and Sustainable Software (GREENS), 2013 2nd International Workshop on. IEEE.

Dingsøyr, T., Nerur, S., Balijepally, V. and Moe, N. (2012), “A decade of Agile methodologies: Towards explaining Agile software development", Journal of Systems and Software, Vol. 85 No. 6, pp.1213-1221.

Dooley, L.M. (2002), "Case study research and theory building", Advances in developing human resources, Vol .4 No. 3, pp.335-354.

Dorst, K. and Cross, N. (2001), Creativity in the design process: co-evolution of problem-solution. Design studies, Vol. 22 No. 5, pp. 425-437.

Fowler, M. and Highsmith, J. (2001), “The Agile manifesto”, Software Development, Vol. 9 No. 8, pp.28-35.

Gonçalves, M. (2017), "Design finds a way: Creative strategies to cope with barriers to creativity", In DS 87-8 Proceedings of the 21 st International Conference on Engineering Design (ICED 17) Vol 8: Human Behaviour in Design, Vancouver, Canada.

Gray, D. (2014), Doing research in the real world. 3rd ed. SAGE, London, pp. 266-293.

Hennessey, B.A. and Amabile, T.M. (2010), “Creativity”, Annual Review of Psychology, Vol. 61, pp.569-598.

Highsmith, J. and Cockburn, A. (2001), “Agile software development: The business of innovation”, Computer, Vol. 34 No. 9, pp.120-127.

James, M. and Walter, L. (2016), Scrum Reference Card. 1st ed. CollabNet Inc.

Jongerius, P. (2012), Get Agile!. Amsterdam: BIS Publ.

Lindsjørn, Y., Sjøberg, D.I., Dingsøyr, T., Bergersen, G.R. and Dybå, T. (2016), "Teamwork quality and project success in software development: A survey of Agile development teams", Journal of Systems and Software, Vol. 122, pp.274-286.

Nonaka, I. (1991), “The knowledge-creating company”, Harvard Business Review, Vol. 69, pp.96-104.

Patton, M.Q. (2002), Qualitative research \& evaluation methods. 3rd ed. SAGE, Thousand Oaks. pp. 339-418.

Sachs, A. (1999), "Stuckness' in the design studio", Design Studies, Vol. 20 No. 2, pp.195-209.

Sutherland, J. and Schwaber, K. (2013), The Scrum Guide.

Wallas, G. (1926), The art of thought. Jonathan Cape, London.

Whitworth, E. and Biddle, R. (2007, August), "The social nature of Agile teams", In: Agile conference (AGILE), 2007. pp. 26-36. IEEE.

Yin, R. (2009), Case study research. 1st ed. SAGE, London. 